\title{
Innovación tecnológica en el ámbito electoral: el caso de la ONPE'
}

Mariano Cucho

mcucho2005@gmail.com

En el presente artículo se hace un recuento de las innovaciones tecnológicas más significativas desarrolladas por la ONPE en los últimos años. Tales innovaciones han sido concebidas bajo el enfoque de gestión por procesos, gestión por resultados, gestión de las competencias y el modelo de excelencia en la gestión de Malcolm Baldrige, el cual se viene implementando desde el año 2014 en la ONPE. Las innovaciones tecnológicas basadas en las Tecnologías de la Información y de la Comunicaciones desarrolladas en la institución buscan mejorar los servicios ofrecidos a la ciudadanía, siguiendo lo establecido en el modelo de excelencia de la gestión. El artículo refleja que, en la actualidad, cada una de estas innovaciones tecnológicas y los efectos positivos que han tenido sitúan a la organización en un alto nivel de competitividad, constituyéndose en un ejemplo para la modernización de otros organismos del sector público en el Perú.

Palabras clave: innovaciones tecnológicas, gestión por procesos, modelo de excelencia, ciudadanía, modernización

This article reviews the most significant technological innovations developed by ONPE in recent years. These innovations were born based on the concepts of process management, results management, competencies management and the Malcolm Bridge performance excellence model implemented at the ONPE in 2014. The technological innovations developed in the institution on the basis of Information and Communication Technologies seek to improve services offered to citizens by following what the excellence in management model establishes. The article reflects that currently each of these technological innovations and their positive effects have yielded a high level of competitiveness in the organization, making it become an example of modernization for other public sector organizations in Perú.

Keywords: technological innovations; process management; model of excellence, citizenship; modernization

1. Este artículo está basado en la ponencia presentada, como parte de la sección Call for Papers, durante el I Congreso Internacional de Ciencias de la Gestión: Gestión de la Innovación e Innovación en la Gestión, desarrollado del 29 de setiembre al 2 de octubre de 2015 en la Pontificia Universidad Católica del Perú por el Departamento Académico de Ciencias de la Gestión y la Facultad de Gestión y Alta Dirección. 


\section{Introducción}

En los últimos tres años, la Oficina Nacional de Procesos Electorales (ONPE) ha desarrollado e incorporado a sus procedimientos un conjunto de innovaciones tecnológicas basadas en las tecnologías de la información y comunicaciones (TIC) con el fin de mejorar los servicios ofrecidos a la ciudadanía, enfatizando una tendencia presente desde su creación en 1995. Estas innovaciones han incidido en las actividades relacionadas con su misión institucional de organizar y ejecutar procesos electorales, pero también se han implementado en diversos procesos internos en el marco de las políticas de calidad establecidas por la Alta Dirección. En consecuencia, se ha posicionado en la ONPE la ejecución de un modelo de excelencia en la gestión que la organización hoy puede socializar como parte de su experiencia institucional en beneficio de los ciudadanos y de otros organismos del sector público peruano.

El objetivo de estas líneas es dar a conocer tales experiencias, centrándose en aquellas que han incorporado tecnología de punta basada en las TIC como parte de cambios tecnológicos mayores en la organización en beneficio de los ciudadanos y de otros organismos del sector público peruano.

Para desarrollar estas innovaciones, la ONPE ha trabajado de forma sistémica bajo el enfoque de gestión por procesos, gestión por resultados y gestión por competencias, siguiendo el modelo de excelencia en la gestión de Malcolm Baldrige (2006). Hay dos pasos o fases comunes a todas estas innovaciones: la adaptación de la estructura orgánica de la ONPE y la planificación estratégica de la innovación.

Respecto de lo primero, hoy la ONPE cuenta con dos unidades orgánicas encargadas de fomentar la innovación y mejora continua: a) la Subgerencia de Innovación y Desarrollo, que propone los lineamientos de política, estándares, buenas prácticas y metodologías para el desarrollo de innovaciones e investigaciones en el ámbito electoral conforme a las políticas nacionales de Gobierno Electrónico; y b) la Subgerencia de Planeamiento, que propone proyectos e iniciativas alineados al Plan Estratégico Institucional (PEI) basados en nuevas tecnologías que contribuyan a la modernización y mejora de los procesos de la ONPE.

Con respecto a la planificación, las innovaciones realizadas en la ONPE se encuentran alineadas con la Política Nacional de Modernización de la Gestión Pública propuesta por la Secretaría de Gestión Pública de la Presidencia de Consejo de Ministros (SGP), plasmada en el DS 004-2013-PCM. Así, para la adecuada gestión institucional, la ONPE se ha alineado con los siguientes ejes estratégicos de la Política de Modernización Institucional: a) nuevo modelo de gestión, b) gestión humana, c) tecnología de punta, d) gestión financiera y de infraestructura, y e) cooperación interinstitucional.

A la fecha, la ONPE ha desarrollado una serie de innovaciones, entre las cuales destacan las siguientes:

a. En cuanto a los servicios ofrecidos a electores y otros actores electorales:

- El voto electrónico presencial (VEP) y el voto electrónico no presencial (VENP)

- $\quad$ El sistema de escrutinio automatizado (SEA) 
- El sistema de cómputo electoral y el software Suite Electoral, que permite contabilizar los votos y emitir los resultados de las elecciones

- El sistema de consulta de miembro de mesa

- La capacitación virtual para actores electorales (PC, HTML5 y APP)

- La verificación de firmas de adherentes para partidos políticos

- El aplicativo para elegir local de votación

b. A nivel interno:

- El sistema de registro de capacitación de actores electorales (SIRCAE), que permite el seguimiento y control de las capacitaciones

- La plataforma ONPEEDUCA y la plataforma virtual de educación electoral en MOODLE para personal de la ONPE y la ODPE

- El sistema de gestión documental, que incluye «la firma digital», permitiendo el ahorro del papel, los recursos físicos y el tiempo, acreditado por INDECOPI

- El sistema de producción de microformas digitales, que garantizan al usuario una mayor confianza y transparencia

- La plataforma Verifondos, que mejoró el proceso de consulta de información sobre fondos partidarios para los usuarios tanto internos como externos a la ONPE

- El sistema de información y seguimiento (SISGOECOR), que permite el seguimiento y control de la gestión electoral en las ODPE a nivel nacional

- El punto de transmisión, utilizado para enviar directamente los resultados electorales desde el local de votación sin tener que trasladarlos al centro de cómputo de la ODPE

- Aplicativos y sistemas informáticos, entre los cuales se pueden mencionar los siguientes:

- Sistema de registro de organizaciones políticas y candidatos

- Sistema de selección de 25 candidatos al cargo de miembros de mesa

- Sistema de georreferenciación para conformación de mesas

- Sistema de control de visitas alineado a las políticas de trasparencia y acceso a la información

Estas innovaciones han permitido que la ONPE obtenga varios reconocimientos en los últimos años, destacándose así su excelencia como organismo público a la vanguardia de la innovación. De particular importancia ha sido la obtención de 16 certificaciones y recertificaciones ISO 9001 de Gestión de la Calidad, Certificación de Ecoeficiencia para tres sedes institucionales y una certificación ISO 27001 en el sistema de gestión de seguridad de la información. Todos estos logros, sin embargo, no podrían haber sido posibles sin un contexto específico en el marco internacional y nacional que ha propiciado la innovación institucional en torno a las nuevas tecnologías. Se trata de un marco de gestión que a su vez tiene una base filosófica, la cual otorga una creciente importancia al saber aplicado a la gestión. 


\section{La innovación y su rol en el sector público}

En el siglo XXI, la gestión de la innovación se ha constituido en un componente clave para la vida y el desarrollo de las organizaciones. Lo que Peter Drucker (1993) definió como la "sociedad del saber" y que hoy llamamos la "sociedad de la información y el conocimiento" es una sociedad donde la acumulación y el manejo de la información se ha constituido en una de las principales variables de la competitividad entre organizaciones, países e individuos. Según Drucker, "ahora el valor se crea mediante la "productividad" y la "innovación", ambas aplicaciones del saber al trabajo" (1993, p. 14). Se trata de un proceso que requiere de la intervención de una nueva cohorte de "trabajadores del saber» dedicados a pensar en nuevas formas de aprovechar el conocimiento en beneficio de la organización y los usuarios.

¿Qué entendemos por innovación? Etimológicamente, lo innovador se refiere a lo "original» o "único». Según el Manual de Oslo, "una innovación es la introducción de un nuevo, o significativamente mejorado, producto (bien o servicio), de un proceso, de un nuevo método de comercialización o de un nuevo método organizativo» (OCDE \& Eurostat, 2006, p. 56), lo cual puede darse en una organización pública o privada.

Aquellas innovaciones que implican mejoras en el servicio propuesto a los clientes son innovaciones de producto. Las que requieren la utilización de métodos, de equipos y/o de unos conocimientos nuevos o mejorados para prestar el servicio son innovaciones de proceso, mientras que si la innovación implica mejoras considerables de las características del servicio prestado y los métodos, equipos y/o conocimientos utilizados para la prestación de un servicio se le llama innovación de producto y de proceso (OCDE \& Eurostat, 2006, p. 64).

Otras clasificaciones ponen énfasis en el papel instrumental de la innovación. Según Morales y otros (2014, pp. 34-35), la evolución del concepto de innovación ha pasado por seis fases: 1) "Como instrumento de cambio», 2) "Como instrumento de industrialización», 3) "Como instrumento de emprendimiento», 4) "Como instrumento de crecimiento económico», 5) "Como instrumento de desarrollo tecnológico», y 6) "Como instrumento de competitividad», correspondiendo la última fase al actual marco de la globalización y el mercado global, donde la innovación resulta de la gestión del conocimiento llevada a cabo por las organizaciones para incrementar su competitividad.

Si bien la noción de "competitividad» ha estado asociada en su origen al sector privado, en los últimos años se ha visto también la importancia de considerarla como parte sustancial del sector público. Los organismos estatales deben ser competentes y competitivos, y ese es uno de los vínculos esenciales con la noción de innovación. Estas innovaciones en el sector público tienen un diferente enfoque: para que la gestión pública funcione bien, es necesario que esta se optimice (SGP-PCM, 2013, p. 5), lo cual requiere implementar y desarrollar los necesarios cambios realizables a través de la gestión de la innovación.

Gestionar la innovación en el sector público supone definir un diseño organizacional y un manejo de recursos que, a diferencia del sector privado, deben pensarse en función del impacto que tendrán en la ciudadanía y en la sociedad. Esto requiere un trabajo de gestión de la innovación enfocado a generar servicios que puedan actuar en beneficio de los electores y demás población hacia la que se dirigen los servicios de la ONPE. 


\section{Gestión de la innovación en la ONPE y la importancia del liderazgo en una institución innovadora}

En la ONPE se promueven, desde la Alta Dirección, diversos mecanismos de innovación orientados a la modernización de la gestión institucional, logrando una mayor eficiencia, eficacia y efectividad en sus procesos. Es así que, en el Plan Estratégico Institucional del año 2014, se incorporaron plenamente lineamientos de gestión dirigidos a incluir las TIC en la ONPE.

Tales lineamientos se alinearon con la Política Nacional de Modernización de la Gestión Pública (PNMGP) desarrollada por la Secretaría de Gestión Pública de la Presidencia del Consejo de Ministros. En estos lineamientos se establecen tres ejes: gobierno abierto, gobierno electrónico y artiluación institucional, los cuales atraviesan cinco pilares de modernización de la gestión pública: 1) políticas públicas, planes estratégicos y operativos; 2) presupuesto para resultados; 3) gestión por procesos, simplificación administrativa y organización institucional; 4) servicio civil meritocrático; y 5) sistema de información, seguimiento, monitoreo, evaluación y gestión del conocimiento.

La ONPE, en su planificación estratégica, definió establecer para cada uno de estos cinco pilares una serie de innovaciones y metas, destacando entre estas aquellas referidas al uso de las TIC. Así, para responder al quinto pilar de la PNMGP, se definió desarrollar específicamente dos puntos: voto electrónico y tecnología de punta. Si bien antes de 2014 la ONPE ya tenía experiencias en el uso del voto electrónico, se trataba de un uso limitado, en solo una circunscripción electoral (distrito de Pacarán, provincia de Cañete, Lima) y con una tecnología aún perfectible. La Alta Dirección decidió por tanto impulsar el desarrollo de esta solución electoral, mejorando la tecnología y ampliando al mismo tiempo la cantidad de circunscripciones en las que esta se implementaría.

Al mismo tiempo, la incorporación de tecnología de punta significó impulsar el desarrollo de innovaciones tecnológicas que incidieran en mejoras para la gestión de la organización, tanto durante los procesos electorales como en el funcionamiento cotidiano de la ONPE. Es a partir de este pilar de modernización que se dio la base para las varias soluciones tecnológicas de gestión interna que hoy la ONPE puede proyectar como logros tangibles de su camino a la innovación.

Sin embargo, realizar cambios en el sector público suele ser más complejo que en el sector privado y para el logro de los cambios tecnológicos en la institución se requirió trabajo en equipo y liderazgo, de lo cual ha quedado testimonio en cada una de las innovaciones tecnológicas desarrolladas por la ONPE en estos últimos años.

\section{Cronología de las innovaciones tecnológicas (1995-2015)}

Desde su puesta en funcionamiento el año 1995, la ONPE contó con un bagaje tecnológico acumulado por el Jurado Nacional de Elecciones, específicamente el uso de un sistema de cómputo para procesar los resultados electorales, el mismo que se empleó por primera vez en las elecciones generales de 1985 (ONPE, 2005, p. 81).

El año 1996, la ONPE comenzó a probar soluciones de voto electrónico, empezando con ensayos controlados y luego con la primera aplicación vinculante de esta 
modalidad de votación en una organización: la Asociación de Padres de Familia (APAFA) del colegio Alfonso Ugarte. Esta modalidad de votación se extendería hasta ser usada durante la segunda vuelta de las elecciones de 2011, en el distrito de Pacarán, provincia de Cañete, Lima. El voto electrónico continuaría desarrollándose en los años venideros y ampliándose la cantidad de circunscripciones que lo pudieran utilizar.

La implementación del voto electrónico estuvo acompañada de un constante mejoramiento del sistema de cómputo electoral usado para el registro de los votos. La más reciente mejora de este sistema se realizó ya en la década del año 2000 con la implementación del software Suite Electoral, el mismo que incorpora una serie de aplicativos para acelerar y facilitar el cómputo de datos. En cuanto a la gestión interna, también se afianzó el año 2010 la implementación de las microformas como mecanismo de soporte a la gestión documental.

Otra innovación cuyo origen se encuentra en el año 2010 es la plataforma Verifondos, elaborada con el fin de acelerar las consultas de ciudadanos y personal de la ONPE sobre el origen de los aportes declarados por los partidos políticos. Esta herramienta fue acogida positivamente tanto por los colaboradores de la ONPE como por la ciudadanía que ahora veía notablemente facilitado su acceso a la información proporcionada por la institución.

El año 2013 fue un hito en la generación de una mayor cantidad de innovaciones trascendentales y dirigidas para los usuarios externos e internos de la ONPE. A fines de ese año se diseñó el sistema de escrutinio automatizado (SEA), que automatiza la fase del escrutinio en la jornada electoral sin modificar el voto convencional o manual en las urnas y que se puso en práctica por primera vez y de forma exitosa en las nuevas elecciones municipales (NEM) de marzo del año 2014. Junto con esta solución, también se ensayó una nueva modalidad de voto electrónico, la cual se aplicó en 2014 y cuya ampliación a todo el país viene proyectándose.

El año 2014 se consolidó a nivel interno la transición de la gestión manual de documentos al novedoso sistema de gestión documental (SGD), aprobado en agosto de 2013. Esto incorporó también la firma digital, que ahora es parte de las gestiones del día a día en la organización. El año 2013 también se concibieron otros proyectos, como la plataforma ONPEEDUCA, así como el inicio de la aplicación institucional de una política de ecoeficiencia.

Desde el año 2013 a la fecha la ONPE ha efectivamente innovado la forma en que se maneja internamente la gestión documental de manera cotidiana. Otras innovaciones vendrían el año 2015, tales como el portal ONPEEDUCA, a través del cual se ofrecen cursos en materia electoral dirigidos a los colaboradores de la ONPE y a ciudadanos interesados por medio de una plataforma virtual amena y didáctica. En la siguiente tabla, se presenta una cronología ordenada del desarrollo de las principales innovaciones con las que hoy cuenta la organización. 
Tabla 1: Cronología de innovaciones ONPE

\begin{tabular}{l|r} 
innovación & año \\
\hline Elige tu local de votación & 2015 \\
Sistema de registro de capacitación de actores electorales (SIRCAE) & 2015 \\
Voto electrónico presencial con tecnología (VENP) & 2014 \\
Sistema de escrutinio automatizado (SEA) y puntos de transmisión & 2014 \\
SISGOECOR & 2014 \\
ONPEEDUCA & 2014 \\
Capacitación virtual para actores electorales & 2013 \\
Sistema de gestión documental y software de firma digital & 2013 \\
Plataforma virtual de educación electoral en MOODLE & 2012 \\
Voto electrónico presencial (módulo) & 2011 \\
Plataforma Verifondos de aportes limpios & 2010 \\
Rediseño del software Suite Electoral & 2006 \\
Software Suite Electoral & 2 \\
\hline & \\
\hline
\end{tabular}

Fuente: ONPE.

Cada una de las innovaciones mencionadas tiene sus especificidades, las cuales presentamos a continuación.

\section{Las innovaciones tecnológicas en detalle}

Las innovaciones desarrolladas por la ONPE pueden clasificarse según si fueron creadas principalmente para actores externos o internos a la organización. Cuando hablamos de "actores externos" nos referimos a los electores, agrupaciones políticas y otros grupos que constituyen a grandes rasgos la ciudadanía y que vienen a ser los principales usuarios de la ONPE, hacia quienes se destina directamente el trabajo de la organización. Por su parte, los "actores internos» son el personal que colabora con la organización para su funcionamiento. Algunas de estas innovaciones pueden ser usadas por ambos tipos de actores indistintamente. Se realizará una descripción general de cada innovación, comenzando por aquellas orientadas a los actores externos a la ONPE y siguiendo con las orientadas a actores internos. 


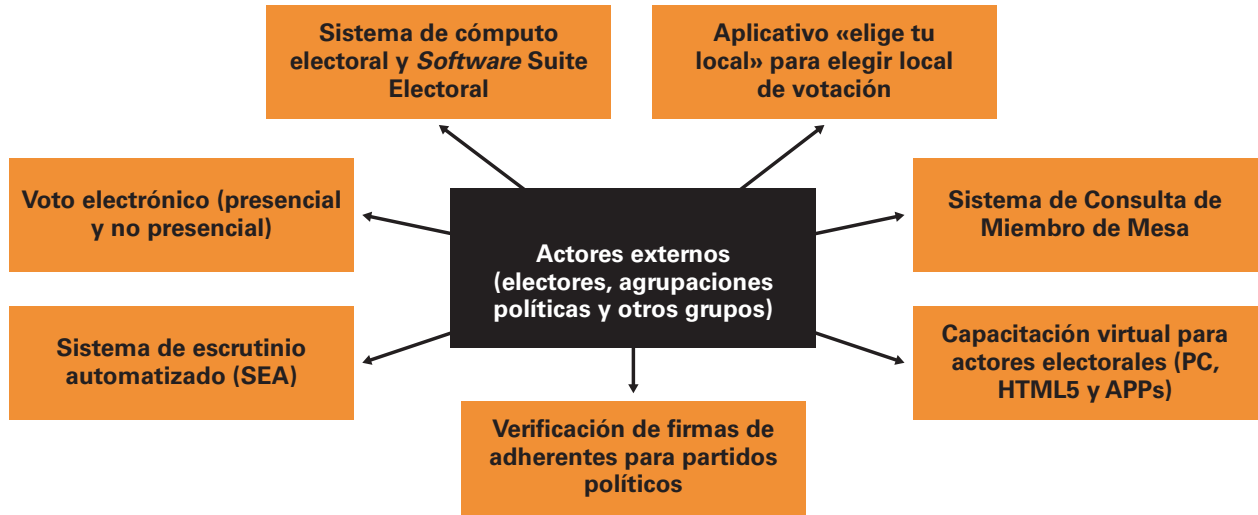

\subsection{Sistema de voto electrónico (presencial y no presencial)}

El voto electrónico es una innovación que la ONPE viene desarrollando casi desde su fundación, remontándose a 1996 la primera experiencia de aplicación vinculante en una organización. Hoy en día se ha avanzado sustancialmente en el esfuerzo de incorporar el voto electrónico dentro de procesos electorales generales en cada vez más circunscripciones.

Hay una distinción entre dos modalidades de voto electrónico: el presencial y el no presencial. El voto electrónico presencial o VEP se refiere a la solución de voto electrónico a ser desplegada a través de algún dispositivo electrónico en un local de votación fijo. Requiere la presencia del elector en el espacio designado para el sufragio y el uso exclusivo de la máquina o dispositivo instalada en este espacio.

El voto electrónico no presencial o VENP, por su parte, podría hacerse desde cualquier equipo conectado a una red -conectado simplemente a Internet- y en el esquema ideal podría facilitar el voto del elector desde la comodidad de su hogar. Se trata por supuesto de una tecnología que resultaría ideal para muchos, pero aún difícil de implementar, incluso en aquellos países con larga y exitosa tradición de uso del voto electrónico. Por lo mismo, el VENP es todavía una tecnología que no se ha aplicado en el Perú en procesos electorales generales, aunque sí en elecciones internas vinculantes, principalmente de organismos públicos y partidos políticos.

EI VEP, en cambio, es la solución de voto electrónico que la ONPE ha intentado implementar en cada vez más procesos electorales generales. La primera aplicación se remonta al año 2011, en la segunda vuelta de las elecciones generales, implementándose la solución tecnológica en el distrito de Pacarán, provincia de Cañete, Lima. Posteriormente, esta solución se volvió a aplicar en Pacarán el año 2013 en dos oportunidades (consulta popular de revocatoria y NEM de 2013), decidiéndose ese año implementar también la solución en el distrito de Santa María del Mar.

Estas pioneras y exitosas experiencias de implementación llevarían a la ONPE a decidir extender el uso del VEP durante las elecciones regionales y municipales (ERM) de 2014, proceso en el cual se decidió usar la tecnología en los siguientes siete distritos: Pacarán (Cañete), Santa María del Mar (Lima), Pucusana (Lima), Punta Hermosa (Lima), Punta Negra (Lima), San Bartolo (Lima) y finalmente el distrito de La Punta (Callao). Este 
sistema también fue empleado en el distrito de Pacarán durante la segunda vuelta regional. La última aplicación de la solución corresponde a finales de 2015 durante las elecciones municipales del distrito de Mi Perú, que se desarrollaron en noviembre de 2015, y se extendió hasta las elecciones generales de 2016. En este último proceso electoral, el voto electrónico se implementó en 19 distritos del ubicados en el departamento de Lima y la Provincia Constitucional del Callao.

Estas experiencias de uso del voto electrónico han ido de la mano con un constante mejoramiento en torno a la tecnología empleada, ya sea del software o de la propia disposición física de los equipos. Así, entre 2011 y 2013, se usó un «módulo» integrado de voto electrónico creado gracias a una asociación entre la ONPE y dos universidades. Para las ERM de 2014 y para las elecciones municipales de 2015, en cambio, se prefirió apostar por una solución consistente en dispositivos flexibles —el módulo no podía desarmarse y, si fallaba alguna de sus piezas, estas no podían reemplazarse sin sustituir todo el equipoque, a su vez, redujeron los costos de transporte y permitieron concentrar el esfuerzo en el desarrollo del software usado, garantizando una cada vez mayor seguridad del mismo en función de los estándares internacionales formulados para el voto electrónico. En las elecciones generales, se proyectó continuar la implementación de esta innovación a través de una nueva plataforma de hardware y software que consistirá en un equipo tablet y dispositivos periféricos (lectora de tarjeta, impresora térmica) y en el nuevo software de voto electrónico.

\subsection{Sistema de Escrutinio Automatizado (SEA)}

El sistema de escrutinio automatizado (SEA) fue usado por primera vez en las NEM del año 2014. Se usó también para las ERM y la segunda elección regional durante el mismo año. Es un software diseñado para automatizar solamente el escrutinio de los votos mediante el uso de la web, siendo una alternativa al voto electrónico, que en teoría automatiza todo el proceso de sufragio. Para ello, el miembro de mesa debe ingresar al nuevo sistema automatizado la información de los votos registrados en su hoja de borrador, tras lo cual se genera el acta de escrutinio (ONPE, 2014, p.105).

Este software hace posible que los datos ingresados de cada mesa sean transmitidos a la sede central, permitiendo tener un continuo monitoreo del avance del escrutinio de cada local de votación y cumpliendo con los estándares de seguridad necesarios para garantizar la transmisión de los datos (2014, p. 105).

Las ventajas de este sistema son la disminución de las actas observadas y la entrega de resultados al $100 \%$ en menos de 24 horas. El porcentaje de actas observadas fue de $0.8 \%$ en las NEM, de $5.7 \%$ en las ERM de 2014 (presidentes y vicepresidentes), de $6.7 \%$ en las ERM de 2014 (consejeros regionales) y de un 1.3\% durante las SER de 2014. Mediante el uso del sistema de escrutinio convencional los porcentajes de actas observadas ascienden a más del $20 \%$. Con respecto a la rapidez del procesamiento de resultados, durante las NEM se entregaron los resultados 16 horas después del cierre de votación, al igual que en el caso de las ERM y las SER.

EI SEA además es un sistema de bajo costo y alto impacto. Su implementación demanda un costo para la institución en la adquisición de equipos informáticos, el mismo 
que es revertido a través de un retorno de la inversión, pues se garantiza la reutilización de los mismos para otros procesos electorales.

\subsection{Sistema de cómputo electoral y el software Suite Electoral}

Para llevar a cabo el cómputo de los resultados, la ONPE emplea el «sistema de cómputo electoral», que a su vez consiste en una red compleja de diferentes procesos entre los cuales destacan los siguientes (2011, p. 6):

- Flujo de actas en el centro de cómputo de la ODPE

- Pruebas internas

- Simulacro de cómputo de resultados

- Procesamiento de resultados

- Digitalización de actas electorales y resoluciones

- Registro de omisos

Todo esto es facilitado a través de la Suite Electoral, que a su vez está compuesta por diferentes aplicativos y softwares usados en cada uno de los procedimientos previamente descritos. En cada proceso electoral la Suite Electoral pasa primero por simulacros de activación y luego por un complejo proceso de apertura, que involucra la apertura de centros de cómputo en cada ODPE, dependiendo de la cantidad de ODPE que se definan para cada proceso electoral.

\subsection{Sistema de consulta de miembro de mesa}

El sistema de consulta de miembro de mesa es un software que forma parte de las soluciones integrales de automatización desplegadas por la ONPE durante un proceso electoral, y permite que el ciudadano consulte a través de Internet si ha sido designado o no como miembro de mesa para determinado proceso electoral. Así, facilita la transmisión de la información a los ciudadanos y que estos puedan prepararse frente a la eventualidad de ser elegidos como miembros de mesa.

\subsection{Capacitación virtual para actores electorales (PC, HTML5 y APP)}

La capacitación electoral es una de las actividades fundamentales en la organización. Consite en preparar a los ciudadanos o, en general, a los actores electorales (electores, personeros y miembros de mesa) para un óptimo desempeño en la jornada electoral, lo cual está establecido por la Ley Orgánica de Elecciones. En virtud de la misión institucional de "garantizar la obtención de la genuina y libre expresión de la voluntad popular para fortalecer la institucionalidad democrática», se requiere que los ciudadanos tengan la información adecuada de acuerdo a los roles que cumplirán el día de la jornada electoral, teniendo que superar los retos propios de nuestro país como la diversidad geográfica, la distribución poblacional, las migraciones y el acceso a la información. 
La ONPE, a través de la Gerencia de Información y Educación Electoral, suscribió el acta de constitución del proyecto denominado Capacitación Virtual para Actores Electorales - Tecnología de Calidad al Alcance de los Peruanos, en el mes de agosto de 2013, para el caso del voto convencional y electrónico.

En el mes de octubre, se implementó y entró en funcionamiento a través de la página web de la ONPE con la finalidad de facilitar de manera ilustrativa e interactiva la comprensión de las tareas y funciones que cumplen los actores electorales, en concordancia con la política de modernización del Estado, que promueve el uso de nuevas tecnologías en beneficio de los ciudadanos.

Si bien el contenido informativo de los módulos de capacitación virtual varía dependiendo de cada proceso electoral, permanece constante la interactividad, definida por una interfaz que facilita el acceso del usuario a la información.

\subsection{Verificación de firmas de adherentes para partidos políticos}

La verificación de firmas es un proceso reglamentado por la ONPE y forma parte de los procesos certificados por el ISO-9660. Esta permite comprobar quiénes son las personas que manifiestan adherirse a una agrupación política al momento de su inscripción. El proceso consiste en la presentación de todos los datos de la agrupación y la lista de sus adherentes por parte de la agrupación política, material entregado tanto en físico como en CD. Una vez en posesión de la ONPE, se usa un software que permite el procesamiento efectivo de los datos, incluyendo la verificación de las huellas dactilares de cada uno de los adherentes. De esta forma, la ONPE puede garantizar que las agrupaciones políticas están siendo inscritas por la libre voluntad de determinados ciudadanos, sin recurrir a suplantación de identidad, falsificación de firmas u otros medios prohibidos.

\subsection{Aplicativo «Elige tu local de votación»}

El recientemente desarrollado aplicativo llamado «Elige tu local de votación» se utilizó por primera vez en las elecciones municipales en el distrito de Mi Perú, en noviembre de 2015. También se usó para la elección del local de votación de los electores de Lima Metropolitana y Callao, así como para las elecciones generales de 2016. Se trata de un aplicativo web que permite al elector elegir su local de votación, estableciendo hasta tres opciones de locales de votación dentro del distrito de residencia declarado en su DNI. Estas alternativas se ordenan de acuerdo a la prioridad establecida por el usuario. El aplicativo permite ingresar los datos y establecer estas prioridades del elector para luego llevar a cabo la asignación final del local. El aplicativo brinda así el camino hacia la solución de una continua preocupación de varios electores: el tener que sufragar en locales de votación muy alejados.

Se presentarán ahora las principales innovaciones orientadas a actores internos de la ONPE, desarrolladas en los últimos años. 

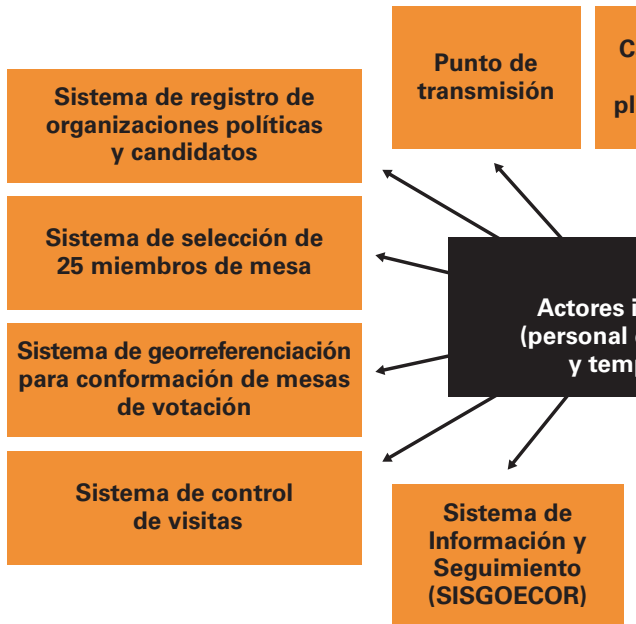

Capacitación virtual ONPEEDUCA en plataforma MOODLE

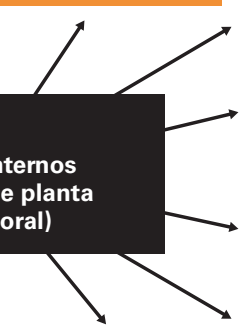

Verifondos -

Sistema de supervisión de fondos partidarios

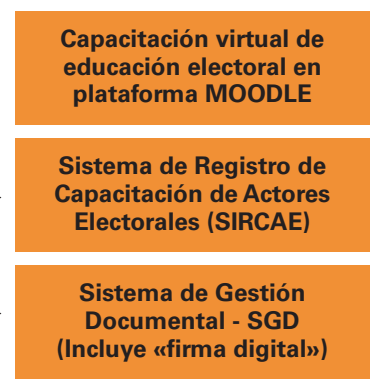

Sistema de Producción de Microformas Digitales

\subsection{El Sistema de Registro de Capacitación de Actores Electorales (SIRCAE)}

Es un sistema que facilita el seguimiento de las acciones de capacitación desarrolladas por la ONPE. De esta forma, se puede saber específicamente a qué actores está alcanzando la capacitación desplegada por la organización y conocer con cuáles se debe hacer mayor esfuerzo.

\subsection{Plataforma ONPEDUCA}

La Gerencia de Información y Educación Electoral de la ONPE suscribió el acta de constitución del proyecto denominado ONPEDUCA, en mayo de 2014, generando conocimiento en los ciudadanos y creando competencias laborales para procesos electorales. El proyecto consiste en una plataforma virtual para la enseñanza y aprendizaje a distancia implementada por dicha gerencia.

La plataforma entró en funcionamiento en el mes de julio del mismo año a través de la web www.onpeduca.edu.pe con la finalidad de facilitar, a través de recursos y medios tecnológicos, una moderna herramienta de capacitación al alcance de todos los peruanos. Incluye también cursos para 22 grupos ocupacionales que ofrece la ONPE mediante la aplicación de un sistema de tutoría y monitoreo permanente, con materiales de autoaprendizaje diseñados de manera ilustrativa e interactiva, para lograr que los participante comprendan tareas y funciones que deberán realizar durante un proceso electoral. A fin de ingresar al campus virtual, cada participante debe contar con un usario y contraseña.

Los principales beneficios que tendrá su implementación en la institución serían los siguientes: 1) ampliación de la cobertura de capacitación para los ciudadanos interesados en postular a cada uno de los 22 grupos ocupacionales que ofrece la ONPE; 2) facilidad de acceso de los ciudadanos al curso virtual a través de una PC, laptop, tablet, iPhone, smartphone o celular con conexión a Internet desde cualquier lugar del país; y 3) gasto cero para la institución en la impresión de material didáctico y en gestión logística, entre otros. 


\subsection{Plataforma virtual de educación electoral en MOODLE para personal de la ONPE y la ODPE}

El área de educación electoral de la GIEE cuenta también con un espacio virtual organizado en la plataforma MOODLE donde se archivan los documentos de capacitación a empleados, los cuales pueden ser consultados por los ciudadanos.

\subsection{Sistema de Gestión Documental y firma digital}

El Sistema de Gestión Documental es un sistema automatizado que funciona por medio de la tecnología de "firma digital» y permite optimizar el proceso de gestión de documentos administrativos de una institución para brindar rapidez, seguridad, mejor control y ahorro. Este sistema emplea nuevas tecnologías libres de licencia, utiliza Internet, no tiene límites territoriales y permite la interoperabilidad con diferentes servicios.

Este sistema se desarrolló en la ONPE en el año 2013 sobre la base de uno desarrollado por RENIEC; sin embargo, el aporte principal del sistema desarrollado por la ONPE es su portabilidad: puede ser usado a nivel nacional a través de cualquier dispositivo electrónico.

Algunos de los beneficios del uso de esta tecnología son los siguientes:

a. Se reduce el uso de papel y el gasto en insumos para la impresión.

b. Se manejan las plantillas de los documentos administrativos de acuerdo a la normatividad de la institución.

c. Se agiliza y mejora notablemente el flujo de las comunicaciones internas y se reduce el tiempo del trámite documentario.

d. Permite garantizar la seguridad y confidencialidad de la documentación.

Adicionalmente a esto, el sistema incorpora la "firma digital», que permite al personal de la ONPE plasmar una rúbrica digital en documentos oficiales sin la necesidad de estar físicamente con el documento. De esta forma el personal que se encuentra lejos de la sede central o que no tiene la posibilidad de recibir un documento impreso de suma urgencia dada su locación puede firmar digitalmente los cargos siempre que cuente con una conexión a Internet y su código de usuario para ingreso al sistema.

\subsection{El Sistema de Producción de Microformas Digitales que garantizan al usuario una mayor confianza y transparencia}

Las microformas digitales son una innovación que viene siendo impulsada por diversos organismos del sector público peruano como medida de protección de datos virtuales. Permite básicamente otorgar a determinados documentos virtuales el mismo valor legal que un documento físico escrito, facilitando de esta forma el archivamiento de la documentación. En la ONPE, la implementación de las microformas se remonta a sus inicios, dado que se trata de una política macro del Estado aprobada desde la primera década de 1990. 


\subsection{Plataforma Verifondos - Aportes limpios}

Verifondos es un novedoso sistema de verificación, control y difusión de la actividad económico financiera de las organizaciones políticas donde se ingresa la información de aportaciones/ingresos semestrales enviadas por las organizaciones políticas. Este sistema fue desarrollado en la ONPE en el año 2010.

El sistema tiene un funcionamiento simple. Primero, las organizaciones políticas presentan su información a la ONPE, siendo cargada a un servidor y luego verificada en una base de datos interna. El procedimiento se resume en la siguiente imagen.

La información recopilada a través de este sistema es difundida en la web institucional a través del módulo de Aportes limpios implementado en el mes de abril del año 2010. En este módulo se pueden efectuar consultas en línea ya sea por aportante, por persona o por candidato de la organización política. Los principales beneficios de la implementación del proyecto de mejora Verifondos - Aportes limpios en la institución son los siguientes:

a. La reducción de tiempo de documentación referida a los aportes de las organizaciones políticas. Se redujo el tiempo de esta búsqueda de aproximadamente cinco horas a un minuto.

b. Difusión inmediata de la información financiera, ya que la información financiera, al estar en formato digital, es registrada automáticamente a la base de datos sin necesidad de enviar documentos internos para su procesamiento. Esta información se visualiza en la página web a través del módulo de Aportes limpios.

c. Simplificación administrativa. Con solo cargar la información de aportaciones al sistema verificación, control y difusión de la actividad económico-financiera de las organizaciones políticas, esta se visualiza inmediatamente en la página web a través del módulo de Aportes limpios.

d. Mejora de los procesos internos de la Gerencia de Supervisión de Fondos Partidarios. Los auditores de esta gerencia han logrado mejorar su productividad y desempeño laboral con apoyo de esta herramienta. Su uso permite que los auditores ahorren tiempo en la elaboración de los informes de verificación de fondos, los cuales son publicados en la web para el acceso de los actores electorales.

\subsection{El punto de transmisión}

El punto de transmisión es otra de las más recientes innovaciones de la ONPE desplegadas durante la jornada electoral. Consiste en la instalación de un equipo dentro de un local de votación desde el cual se pueden transmitir los resultados directamente a la sede central, sin tener que trasladarlos hasta el centro de cómputo de la ODPE, ahorrándose de esta forma una considerable cantidad de tiempo. Los puntos de transmisión se emplearon por primera vez en algunos locales de votación durante las ERM de 2014 y también se incorporaron durante las EG de 2016. 


\subsection{Sistema de georreferenciación para conformación de mesas}

Otra innovación de suma utilidad que ha contribuido a expandir la llegada de la ONPE hacia los electores de las zonas más alejadas del país, así como a simplificar la labor de los colaboradores de la organización, es el sistema de georreferenciación para conformación de mesas. Se trata de un sistema que depende a su vez del Sistema de Información Geográfico Electoral (SIGE), el cual se ha implementado en ONPE desde el año 2009.

La información del SIGE permite que a través del sistema de georreferenciación se identifique cada tipo de circunscripción electoral, con su respectiva densidad demográfica de electores, permitiendo una asignación racional de locales de votación según las posibilidades reales de los electores para acceder a los mismos. Para ello se hace uso de criterios de cercanía, accesibilidad y personal disponible, entre otros aspectos. Gracias a este sistema, hoy en día es posible garantizar que los peruanos de las zonas más recónditas del país puedan ejercer su derecho al voto.

\section{La gestión de la innovación y la gestión de calidad en la institución}

En la ONPE la gestión de la innovación está ampliamente ligada al desarrollo de diversas políticas de calidad. Como parte de estas políticas, la institución tiene los siguientes pilares:

1) Reafirmar los valores democráticos, brindando a la ciudadanía, a las organizaciones políticas y a la sociedad civil servicios acorde a sus requerimientos, garantizando en su prestación el respeto a los principios de igualdad, transparencia y estricto apego legal.

2) Mejorar continuamente nuestros procesos internos contando para ello con personal calificado y comprometido con la mejora continua, así como con la tecnología que permita asegurar la eficacia del Sistema de Gestión de Calidad.

Es por ello que 16 procesos de la institución cuentan con la certificación ISO 9001, tres sedes con la Certificación de Ecoeficiencia, y un proceso con la certificación ISO 27001. La ONPE se encuentra alineada a una política de ecoeficiencia que a la fecha ya se encuentra debidamente certificada. Todas las mejoras en gestión de calidad de los procesos de la institución están orientadas por el respeto al medioambiente y el uso adecuado y racional de los recursos físicos. Mantener estos estándares de calidad requiere sostener las innovaciones ya señaladas y mejorarlas continuamente, cuidando que los procesos internos continúen de forma eficiente y ordenada, siempre con el usuario final y su comodidad en mente.

De otro lado, las 5 S es un método que busca generar una mayor productividad de los colaboradores de una organización a partir de la implementación de pautas para lograr ambientes de trabajo más limpios, seguros y ordenados que permitan el ahorro de tiempo y costos. Esta práctica de calidad tiene las siguientes etapas, constituyendo cada etapa una "S», que representa su significado en japonés: 1) Seiri: clasificar las cosas necesarias y las innecesarias; 2) Seiton: arreglar los elementos para que puedan 
ser fácilmente encontrados; 3) Seiso: limpieza del lugar de trabajo; 4) Seiketsu: implica mantener el lugar de trabajo cómodo y productivo; y 5) Shitsuke (disciplina): convertir en hábito las pautas previamente descritas. Esta última «S» implica un cambio cultural dentro de la organización. En la ONPE, esta práctica se encuentra alineada a otras estrategias de calidad, como por ejemplo la ecoeficiencia.

\section{Lecciones aprendidas replicables en otras organizaciones públicas}

Hoy en día, la experiencia institucional de la gestión de la innovación en la ONPE sirve de referente para otros organismos públicos que se encuentran implementando políticas de innovación tecnológica y de calidad, contribuyendo así a un empoderamiento de la competitividad de los organismos públicos del Perú y encaminándolos hacia un modelo de excelencia en la gestión pública como el que actualmente se reproduce en la ONPE.

Esto se ve, por ejemplo, en la incorporación de tecnologías orientadas a obtener una gestión "cero papeles» por parte de algunos organismos públicos que han solicitado a la ONPE jornadas de capacitación y asesoría técnica para el desarrollo de sus propios sistemas de gestión documental. Es el caso del programa "Juntos» del Ministerio de Desarrollo e Inclusión Social, para el cual se han programado jornadas de capacitación durante el mes de setiembre de 2016 en las que el personal de la ONPE socializará su experiencia, de forma que "Juntos" pueda también crear su propio sistema de gestión documental. Aquí se aprecia el impacto positivo que la innovación tecnológica de la ONPE puede tener no solo para el mejor desempeño de su misión institucional, sino para estimular a otros organismos del Estado peruano a replicar buenas prácticas de gestión, ubicándose así como una organización de vanguardia de la modernización de la gestión pública en el Perú. 


\section{bibliografía}

\section{Camisón, César; Tomás}

Gonzales \& Sonia Cruz

2006 Gestión de la calidad: conceptos, enfoques, modelos y sistemas.

Madrid: Pearson.

\section{Drucker, Peter}

1993 La sociedad poscapitalista. Buenos Aires: Sudamericana.

\section{Hirano, Hiroyuki}

5 Pillars of the Visual Workplace. Portland, Oregon: Productivity Press.

\section{ISO}

2008 Norma internacional, traducción oficial. Sistemas de gestión de la calidad. Ginebra: Secretaría General de ISO.

\section{Michalska, J. \& D. Szewieczek}

"The 5S methodology as a tool for improving the organization". Journal of Achievements in Materials and Manufacturing Engineering, 2 (24), pp. 211-214.

\section{Morales, Oswaldo; Ángel Barrera, Milagros Rodríguez, Carla Romero \& Rosa Távara} los gobiernos locales del Perú. Lima: Universidad ESAN.

\section{OCDE \& Eurostat}

2006

Manual de Oslo: guía para la recogida e interpretación de datos sobre innovación (tercera edición). Madrid: Grupo Tragsa.

\section{ONPE}

2005

Los procesos electorales en el Perú: 19781986. Problemas y lecciones. Lima: ONPE.

\section{ONPE}

El voto electrónico en la práctica: perspectivas y dinámicas desde la experiencia de las elecciones regionales y municipales 2014. Lima: ONPE.

\section{ONPE}

Reporte de procesos y consultas 11. Lima: ONPE.

\section{PRAXIS}

Modelo de excelencia en la gestión Malcolm Baldrige (traducción libre por José Antonio Villagra Villanueva). Disponible en: $<$ http://www.praxis.com.pe/portal/sites/ default/files/m baldrige 2006.pdf $>$.

\section{Secretaría de Gestión Pública de la PCM}

Plan de implementación de la Política Nacional de Modernización de la Gestión Pública 2013-2016. Lima: Presidencia del Consejo de Ministros.

Fecha de recepción: 16/03/2016

Fecha de aceptación: 26/09/2016 